\title{
Boquillas de Abanico Plano de Amplio Espectro y por Aire Inducido en la Eficacia de Glifosato y Paraquat
}

\author{
Abelino Pitty ${ }^{1}$, Elvis Ernesto Barrios ${ }^{2}$ y Rommel Reconco ${ }^{3}$
}

\begin{abstract}
Resumen. Se evaluó el control de malezas aplicando glifosato con boquillas de abanico plano de amplio espectro (XR $11003 \mathrm{VS}$ ) y de aire inducido (AI 11003VS) y se usó paraquat con boquillas abanico plano de amplio espectro (XRC 11003VS) y por aire inducido (AIC 11003VS). Con papel hidrosensible se determinó la distribución y tamaño de las gotas con las boquillas AI 11003VS y XR 11003VS aplicando 250 y $165 \mathrm{~L} / \mathrm{ha}$. La boquilla de aire inducido tuvo gotas más grandes que la de amplio espectro; al bajar el volumen de 250 a 165 L/ha disminuyó la cobertura con ambas boquillas. En campo se determinó la efectividad de las boquilla de aire inducido (AI 11003VS) y de amplio espectro (XR 11003VS) con glifosato; y la efectividad de la boquilla de aire inducido (AIC 11003VS) y de amplio espectro (XRC 11003VS) con paraquat. El diseño estadístico fue de bloques completamente al azar con seis réplicas. Con glifosato se utilizó la dosis recomendada de $890 \mathrm{~g} / \mathrm{ha}$ de ingrediente activo, y al 75\% de esa dosis (668 g/ha); para paraquat se usó la dosis de $500 \mathrm{~g} / \mathrm{ha}$ de ingrediente activo y al $75 \%$ de esa dosis (375 g/ha). Se determinó el control sobre las malezas pasto estrella (Cynodon nlemfuensis Vanderyst) con glifosato, y con paraquat con pangola (Digitaria spp.). Con glifosato no hubo diferencia estadística en la interacción boquilla por dosis, ni tampoco entre las boquillas de amplio espectro y aire inducido. Hubo reducción en el control al reducir el glifosato de $890 \mathrm{~g} / \mathrm{ha}$ de ingrediente activo a $668 \mathrm{~g} / \mathrm{ha}$. Con paraquat no se encontró diferencia estadística en la interacción boquilla por dosis, ni tampoco entre las boquillas por aire inducido y amplio espectro. En la primera evaluación con las dosis de paraquat no se encontró diferencia estadística, pero en la segunda y la tercera hubo menor control al bajar las dosis de 500 $\mathrm{g} / \mathrm{ha}$ de ingrediente activo a $375 \mathrm{~g} / \mathrm{ha}$. No hubo variación entre boquillas en el control de malezas, con ninguna de las dosis.
\end{abstract}

Palabras clave: Cynodon nlemfuensis, Digitaria spp., volumen de aplicación, tamaño de la gota.

\section{Effectiveness of Extended Range Flat Fan Nozzles and Air Induction Nozzles with Glyphosate and Paraquat}

\begin{abstract}
Weed control was evaluated applying glyphosate with extended range flat fan nozzles (XR 11003VS) and air induction flat fan nozzles (11003VS AI). In another experiment, paraquat was used with extended range flat fan nozzles (11003VS XRC) and air induction flat fan nozzles (AIC 11003VS). With water sensitive paper was determined the distribution and droplet size of the XR 11003VS and AI 11003VS nozzles applying 250 and $165 \mathrm{~L} / \mathrm{ha}$. The air induction nozzle had larger droplets than the extended range. Decreasing the application volume from 250 to $165 \mathrm{~L} / \mathrm{ha}$ decreased coverage with both nozzles. In field trials, the effectiveness of the air induction (AI 11003VS) and extended range nozzles (XR 11003VS) with glyphosate was determined, also the efficacy of the AI (AIC 11003VS) and extended range nozzles (XRC 11003VS) with paraquat. We used a factorial experiment arranged in a randomized complete block design with six replicas. Glyphosate was applied at the recommended active ingredient dose of $890 \mathrm{~g} / \mathrm{ha}$ and $75 \%$ of the recommended dose (668 g/ha); with paraquat the recommended dose was $500 \mathrm{~g} / \mathrm{ha}$ of active ingredient and $75 \%$ of the recommended dose $(375$ $\mathrm{g} / \mathrm{ha}$ ). We determined the percentage of visual damage on African Bermuda grass (Cynodon nlemfuensis) with glyphosate, and crabgrass (Digitaria spp.) with paraquat. With glyphosate there was no statistical difference in the interaction nozzle by dose, or between nozzle types. There was a decrease in weed control when the glyphosate application rate was reduced from $890 \mathrm{~g} / \mathrm{ha}$ of active ingredient to $668 \mathrm{~g} / \mathrm{ha}$. With paraquat there was no statistical difference found for the interaction of nozzle by dose, or between the two types of nozzles. There was no statistical difference between the doses of paraquat in the first evaluation, but in the second and the third there was less weed control when dose was reduced from $500 \mathrm{~g} / \mathrm{ha}$ of active ingredient to $375 \mathrm{~g} / \mathrm{ha}$. There was no difference in the weed control between nozzles with any of the doses.
\end{abstract}

Keywords: Application volume, Cynodon nlemfuensis, Digitaria spp., droplet size, herbicide.

\footnotetext{
${ }^{1}$ Escuela Agrícola Panamericana, Departamento de Ciencia y Producción Agropecuaria, Zamorano, Honduras, correo electrónico apitty@zamorano.edu

${ }^{2}$ Panamá, Panamá, correo electrónico e2b2_1988@hotmail.com

${ }^{3}$ Escuela Agrícola Panamericana, Carrera de Agronegocios, Zamorano, Honduras, correo lectrónico rreconco@zamorano.edu
} 


\section{Introducción}

El éxito del control de malezas con herbicidas no depende únicamente del herbicida; existen otros factores de igual importancia que en muchas ocasiones no son tomados en cuenta. Muchos problemas que se presentan al utilizar herbicidas se deben a fallas de los usuarios, ya sea por desconocimiento del manejo, por formas inadecuadas de aplicación, o por deficiencia en el mantenimiento de los equipos. Con respecto a esto hay que tomar en cuenta: la calibración, la presión, la velocidad, la agitación en el tanque, el aguilón, las boquillas y sus filtros, y algunas normas de mantenimiento (Doll 1981).

La efectividad de los herbicidas está fuertemente influenciada por el tipo de boquilla y el volumen de agua usado, además de las condiciones ambientales (Hatterman-Valenti et al. 2006), presencia de suelo en las hojas (Zhou et al. 2006) y la calidad de agua (Gómez Vargas et al. 2006). Por lo tanto, se debe ser eficiente al momento de aplicarlos para evitar reducción en la efectividad (Thornhill y Matthews 1994).

Todos los equipos de aplicación tienen tres características en común, el líquido para asperjar está contenido en un depósito (el tanque de aspersión), del cual es movido por bomba de presión o por un sistema alimentado por gravedad a las boquillas. Una boquilla es la terminación de un tubo a través de la cual el líquido puede salir en forma de un chorro. Por lo tanto, la boquilla es una de las partes más importante de la aspersora, pero a menudo se le descuida y con poca frecuencia se revisa para asegurarse que están aplicando correctamente (Matthews 1979). Las boquillas pueden ser simples o extremadamente complejas en su construcción. Independientemente del costo, complejidad o precisión, todas las boquillas regulan el flujo del líquido y producen un espectro de aspersión específico (Tharrington y Tate 1976).

Los ángulos de aspersión usados en las aplicaciones de herbicidas son de 65,80 y $110^{\circ}$. Cada ángulo de aspersión requiere una altura diferente de la barra o el aguilón para un mismo espaciamiento entre boquillas, si se quiere obtener uniformidad. Los ángulos menores requieren una ubicación más elevada de la barra o aguilón y obligan a las gotas a recorrer mayores distancias antes de impactar sobre su objetivo. Por lo tanto, influencias externas como la temperatura, la humedad y el viento tienen mayor oportunidad para influir sobre el patrón de aspersión (Tharrington y Tate 1976, Rosses y Urrutia 1999).

Las funciones de las boquillas son las de dividir el líquido en gotas, formar el patrón de aspersión, y controlar el flujo del líquido. La desintegración aleatoria de la lámina de aspersión que surge del orificio de la boquilla produce un amplio espectro de gotas. Se obtiene una distribución uniforme cuando se usa más de una boquilla, mediante el traslape de los bordes adelgazados de las boquillas individuales. La calidad de aspersión, o rango del tamaño de las gotas, se hace más fina a medida que el tamaño del orificio de la boquilla se reduce, aumenta el ángulo de la boquilla y la presión de la aplicación. Inversamente, las gotas de agua son más grandes al aumentar el diámetro del orificio, y se reduce el ángulo de la boquilla y la presión de aplicación (Labrada et al. 1996).

Las boquillas asistidas por aire son un nuevo tipo de boquilla, que a diferencia de las otras boquillas, combinan aire con el líquido a aplicar formando gotas gruesas y grandes llenas de aire, reduciendo el número de gotas que son susceptibles a la deriva. Esta particularidad se debe a que posee un orificio que funciona como venturi o aspirador de aire, que absorbe aire hacia el interior de la boquilla donde se mezcla con el líquido que se desea aplicar (Spraying Systems Co. 2004).

Las boquillas de abanico plano de amplio espectro son las más usadas debido a que proporcionan una excelente distribución de la pulverización dentro de un rango amplio de presiones; cuando son operadas a presiones bajas la deriva tiende a disminuir y con altas presiones logra una mejor cobertura (Spraying Systems Co. 2004).

Este estudio se realizó con el objetivo de determinar la efectividad de los herbicidas glifosato y paraquat con las boquillas de abanico plano por aire inducido (AI110 03VS) y de amplio espectro (XR110 03VS) y con dos volúmenes de agua. 


\section{Materiales y Métodos}

Estimación de la cobertura de las boquillas. Se comparó la boquilla de abanico plano de amplio espectro (XR 11003VS) con la de aire inducido (AI 11003VS), ambas con un ángulo de aplicación de $110^{\circ}$, un caudal de 0.3 galones por minuto a a presión de $2.76 \mathrm{~kg} / \mathrm{cm}^{2}$ (40 psi), de acero inoxidable y fabricadas por Teejet ${ }^{\circledR 4}$. Se colocaron sobre el suelo seis tarjetas hidrosensibles fabricadas por Syngenta Crop Protection $(52 \times 76 \mathrm{~mm})$ en dos líneas rectas (tres tarjetas en cada línea) separadas $150 \mathrm{~cm}$, las tarjetas en cada línea estaban separadas $200 \mathrm{~cm}$.

El agua se aplicó con una bomba de mochila modelo $\mathrm{T}^{5}$, equipada con un tanque de acero inoxidable presurizado con $\mathrm{CO}_{2}$. Los dos tipos de boquillas se colocaron en el mismo aguilón, pero separadas $150 \mathrm{~cm}$, esto permitió aplicar con las dos boquillas al mismo tiempo y tener la misma velocidad de aplicación y presión para ambas. Las aplicaciones se hicieron a $2.10 \mathrm{~kg} / \mathrm{cm}^{2}$ (30 psi); se aplicaron 165 $\mathrm{L} / \mathrm{ha}$ a una velocidad de $6.5 \mathrm{~km} / \mathrm{h}$; la aplicación a 250 L/ha se hizo a $4.32 \mathrm{~km} / \mathrm{h}$. La altura de aplicación fue $40 \mathrm{~cm}$ sobre las tarjetas.

Después de aplicar el agua, de cada tarjeta se tomó una foto a colores. El porcentaje de cobertura se determinó analizando la foto de cada tarjeta con el programa ArcGIS. Se clasificó un color falso basado en la cantidad de pixeles de cada foto para identificar un umbral y calcular el área cubierta por las gotas de agua. El umbral mínimo establecido fue de 140 pixeles, así que cualquier valor menor de 140 se consideró afectado por la gota de agua. El color falso se convirtió a un polígono o puntos en ArcGIS para determinar el total de pixeles en cada foto. Esto se convirtió a una nueva imagen usando solamente los valores menores de 140 pixeles. Para calcular el porcentaje de cobertura en cada foto, se dividió el número de pixeles (menor de 140) entre la cantidad de pixeles totales en la foto de cada tarjeta, multiplicado por 100 .

\footnotetext{
${ }^{4}$ Spraying Systems Co., Wheaton, Illinois, Estados Unidos.

${ }^{5}$ Fabricada por R \& D Sprayers, Opelousas, Louisiana, Estados Unidos.
}

El diseño estadístico fue de bloques completamente al azar con tres réplicas, se analizó como un factorial de $2 \times 2$. Los factores analizados fueron el tipo de boquilla y el caudal de agua aplicado. Los datos se analizaron con el SAS (SAS 9.1) con PROC GLM para los análisis de varianza y Duncan para la separación de medias con un nivel de significancia de $5 \%(\mathrm{P} \leq 0.05)$.

Eficacia del glifosato. Un terreno infestado con la maleza perenne Cynodon nlemfuensis Vanderyst (pasto estrella) se dividió en 24 lotes de $4 \mathrm{~m}$ de ancho y $6 \mathrm{~m}$ de largo. Se aplicó glifosato (formulación Inquigrass 35.6 $\mathrm{SL}^{6}$ ) a la dosis recomendada de 890 $\mathrm{g} / \mathrm{ha}$ de ingrediente activo (100\%), y a $668 \mathrm{~g} / \mathrm{ha}(75 \%$ de la recomendada en la etiqueta). Se aplicó con una bomba de mochila modelo $\mathrm{T}$, equipada con un tanque de acero inoxidable, presurizada con $\mathrm{CO}_{2}$; el aguilón era de $2 \mathrm{~m}$ de ancho con cuatro boquillas separadas cada $50 \mathrm{~cm}$. La aplicación se hizo de 8 a 11 de la mañana, en un día soleado, a una velocidad de 4.32 $\mathrm{km} / \mathrm{h}$, una presión de $2.10 \mathrm{~kg} / \mathrm{cm}^{2}$ (30 psi), y a una altura de $40 \mathrm{~cm}$ sobre las malezas. Se utilizaron las boquillas Teejet ${ }^{\circledR}$ XR 11003 VS y AI 11003 VS.

Los datos fueron tomados a los 11, 18 y 25 días después de la aplicación. Se tomó el daño a la maleza expresado en porcentaje, dos personas hicieron las evaluaciones y se tomó el promedio de las dos evaluaciones. El porcentaje de control se determinó comparando cada lote con un testigo que no fue aplicado con glifosato ni se usó en al análisis estadístico. La escala visual de daño sobre el pasto estrella era $0 \%$ cuando la aplicación no tuvo efecto, 10 a $30 \%$ un efecto ligero, 40 a $60 \%$ efecto mediano, 70 a $90 \%$ efecto severo y $100 \%$ muerte total (De la Cruz 1986). El porcentaje de control de maleza no fue transformado al arcoseno ya que tenían una distribución normal porque todos los valores estaban alrededor de 50\% (Steel y Torrie 1980).

El diseño estadístico fue de bloques completamente al azar con seis réplicas, se analizó como un factorial de $2 \times 2$. Los factores analizados fueron el tipo de boquilla y la dosis de glifosato. Los datos se analizaron con el Statistical Analysis System

\footnotetext{
${ }^{6}$ Fabricado por Industria Química S.A., Costa Rica.
} 
(SAS 9.1) con el modelo general de modelos lineales, para los análisis de varianza y la separación de medias de Duncan se usó con un nivel de significancia de 5\% $(\mathrm{P} \leq 0.05)$.

Eficacia del paraquat. El estudio fue con la maleza predominante Digitaria spp. (pangola), gramínea anual. El terreno se dividió en parcelas de $4 \times 4 \mathrm{~m}$, se utilizaron las boquillas de rango extendido Teejet XRC 11003VS y aire inducido AIC 11003VS. Estas boquillas tienen un patrón de aplicación, descarga y tamaño de gotas igual a las usadas en el estudio con glifosato, pero el cuerpo de la boquilla trae incorporado una arandela de calce, lo que asegura el sellado con el aguilón.

Se utilizó como paraquat el Gramoxone ${ }^{\circledR}$ Super $\mathrm{SL}^{7}$ (ingrediente activo a $200 \mathrm{~g} / \mathrm{L}$ ). Se utilizó la dosis recomendada en la etiqueta de $500 \mathrm{~g} / \mathrm{ha}$ de ingrediente activo y $375 \mathrm{~g} / \mathrm{ha}$ (75\% de la dosis recomendada en la etiqueta). La aplicación se hizo con la bomba de mochila modelo $\mathrm{T}$ descrita en el estudio con glifosato. La aplicación se hizo de 8:35 a 9:00 de la mañana, en un día soleado, a una velocidad de $4.9 \mathrm{~km} / \mathrm{h}$, una presión de $2.81 \mathrm{~kg} / \mathrm{cm}^{2}$ (40 psi) y una altura sobre las planta de $40 \mathrm{~cm}$.

Los datos fueron tomados a los 3, 8 y 15 días después de la aplicación. Se tomó el daño a la maleza expresado en porcentaje, siguiendo la metodología descrita en el experimento con glifosato. El porcentaje del control de Digitaria spp. no fue transformado al arcoseno ya que tenían una distribución normal porque los valores estaban alrededor de $50 \%$ (Steel y Torrie 1980).

El diseño estadístico fue en bloques completamente al azar con seis réplicas, se analizó como un factorial de $2 \times 2$. Los factores analizados fueron el tipo de boquilla y la dosis de paraquat. Los datos se analizaron con el Statistical Analysis System (SAS 9.1) con el modelo general de modelos lineales, para los análisis de varianza y la separación de medias de Duncan se usó con un nivel de significancia de 5\% $(\mathrm{P}<0.05)$.

\footnotetext{
${ }^{7}$ Fabricado por Syngenta.
}

\section{Resultados y Discusión}

Estimación de la cobertura y visualización del tamaño de las gotas. Hubo diferencia estadística en la cobertura entre los dos tipos de boquilla y los dos volúmenes de aplicación; pero no hubo interacción entre los dos factores (Figura 1). La cobertura con la boquilla AI 11003VS fue $18.9 \%$ y con XR 11003 VS fue $14.5 \%$. Cuando se aplicaron $250 \mathrm{~L} / \mathrm{ha}$, la cobertura fue $20.3 \%$ y con $165 \mathrm{~L} /$ ha se redujo a $13.2 \%$.

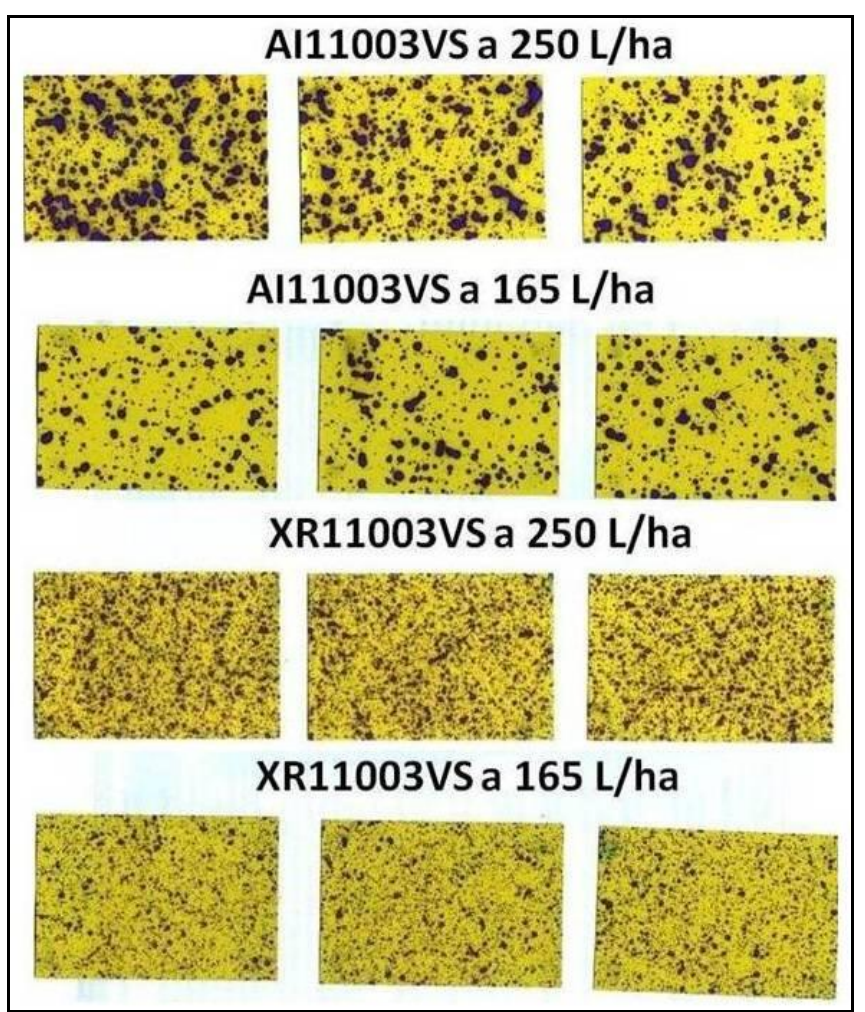

Figura 1. Distribución de gotas de agua con las boquillas XR11003VS (amplio espectro) y AI11003VS (aire inducido), con dos volúmenes de aplicación: $165 \mathrm{~L} / \mathrm{ha}$ aplicado a $6.5 \mathrm{~km} / \mathrm{h}$ y $250 \mathrm{~L} / \mathrm{ha}$ aplicado a $4.32 \mathrm{~km} / \mathrm{h}$.

La mayor cobertura obtenida con la boquilla AI 11003 VS, comparada con la XR 11003VS, puede aumentar la efectividad de los herbicidas de contacto (paraquat o bentazón) que necesitan cubrir las hojas 
para tener un buen control de malezas; lo que no es indispensable con herbicidas sistémicos (glifosato, 2,4-D o fluazifop). La mayor cobertura fue con 250 L/ha debido a que se usa más agua por hectárea, lo que también debe aumentar el control de malezas con herbicidas de contacto (Knoche 1994).

La boquilla AI11003VS tuvo mayor cantidad de gotas grandes a extra grande, comparado con la XR11003VS (Figura 1). Esto se debe a la mezcla del agua con aire que causa la boquilla, que hace que a presiones de 2 bares (29 psi o $2.00 \mathrm{~kg} / \mathrm{cm}^{2}$ ) o a 2.5 bares $\left(36\right.$ psi $\left.02.48 \mathrm{~kg} / \mathrm{cm}^{2}\right)$ tenga gotas extremadamente grandes y de 3 bares (44 psi o 3.04 $\left.\mathrm{kg} / \mathrm{cm}^{2}\right)$ a 5.5 bares $\left(80\right.$ psi o $\left.5.52 \mathrm{~kg} / \mathrm{cm}^{2}\right)$ gotas grandes (Spraying Systems Co. 2004). A esto se debe la característica antideriva de la boquilla, lo que la hace más eficiente aplicando con velocidad del viento alta.

Hubo mayor cantidad de gotas finas con la XR11003VS (Figura 1). Esto se debe a su diseño, hecho para tener una amplia cobertura, obteniendo gotas finas con presiones de 2 bares (29 psi) a 4 bares (58 psi o $40 \mathrm{~kg} / \mathrm{cm}^{2}$ ) (Spraying Systems Co. 2004). Las gotas pequeñas dan mayor cobertura, pero en aplicaciones con viento fuerte habrá mayor deriva, comparado con la AI11003VS, lo que puede reducir la eficacia de los herbicidas.

Eficacia del glifosato. En ninguna de las tres evaluaciones hubo diferencia estadística en la interacción boquilla por dosis del glifosato, lo que indica que los efectos de la boquilla y dosis son independientes. El control con las dos boquillas no depende de la dosis, ni el control con las dos dosis depende del tipo de boquilla usado.

En las tres evaluaciones, el control fue igual con las dos boquillas (Figura 2). Esto indica que para aplicar glifosato se puede usar la boquilla AI110 03VS o la XR110 03VS sin afectar el control del pasto estrella. Otros estudios con glifosato tampoco encontraron diferencia en el control de malezas con estas boquillas, a pesar de que documentaron que el tamaño de las gotas es diferente (Jones et al. 2002, Feng et al. 2003). Lo anterior puede ser debido a que el glifosato es sistémico y se puede mover a través de la planta, lo que compensa por la poca cobertura del follaje (Venturelli et al. 2006, Knoche et al. 1994). Ya que el tipo de boquilla evaluado no influye en el control, se puede usar la boquilla que esté más disponible o la más económica, incluso se pueden mezclar.

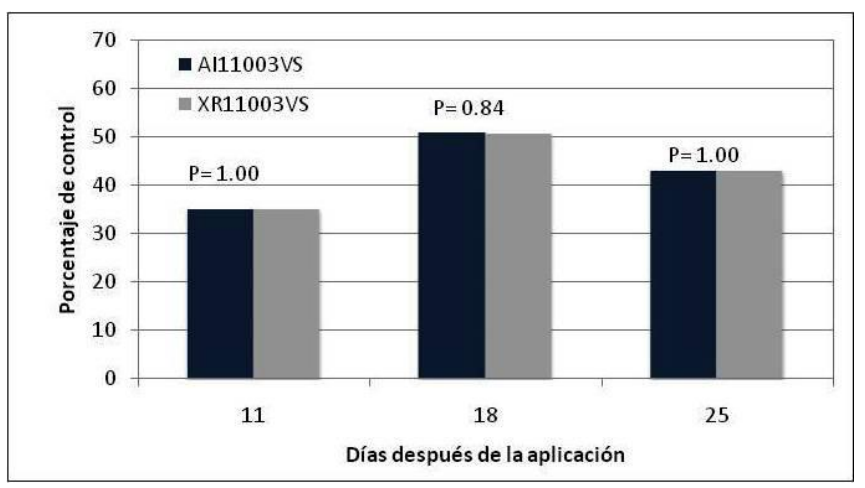

Figura 2. Porcentaje de control de pasto estrella (Cynodon nlemfuensis) con las boquillas de aire inducido (AI 11003VS) y amplio espectro (XR $11003 \mathrm{VS})$, a los 11,18 y 25 días después de la aplicación de glifosato. Los valores sobre la barra son la probabilidad en cada evaluación e indican que no hay diferencia entre tratamiento.

En la dosis hubo diferencia estadística $(\mathrm{P} \leq 0.01)$ en el control del pasto estrella en las tres fechas de muestreo (Figura 3), al bajar la dosis de 890 a 668 $\mathrm{g} /$ ha de ingrediente activo (equivale a $75 \%$ de la dosis recomendada). A los 11 días después de la aplicación el control fue de 38 y 33\%, con dosis de 890 y 668 $\mathrm{g} /$ ha de ingrediente activo, respectivamente; a los 18 días de 54 y $48 \%$ y a los 25 días el control fue 46 y $40 \%$. Esto indica que no se puede reducir la dosis del herbicida porque disminuirá el control sobre el pasto estrella, y que a la dosis usada y el tamaño de la maleza el glifosato no es efectivo para controlar el pasto estrella.

Hubo una tendencia de subir el control del pasto estrella en la segunda evaluación y disminuir en la tercera (Figura 2 y 3 ). Esto puede ser debido a que en la primera evaluación el herbicida no había hecho su efecto completo sobre el pasto estrella y en la tercera 
evaluación la maleza comenzaba a rebrotar, por lo que se redujo el daño.

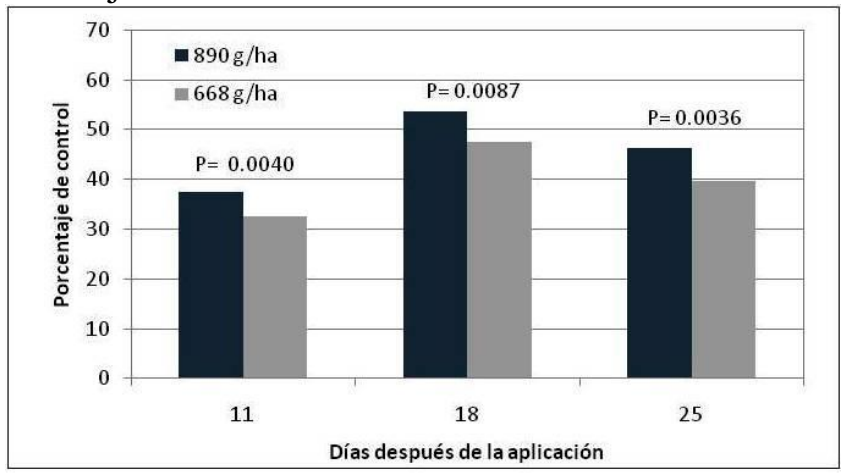

Figura 3. Porcentaje de control del pasto estrella (Cynodon nlemfuensis) con 890 y 668 g/ha de ingredientes activo de glifosato (promedios de las dos boquillas). Los valores sobre las barras indican la probabilidad en cada evaluación.

Eficacia del paraquat. La interacción de la boquilla por la dosis de paraquat no produjo diferencia significativa en el control de Digitaria spp., lo que indica que estos factores son independientes. Las boquillas tampoco presentan diferencia significativa en ninguna de las evaluaciones (Figura 4). Esto indica que bajo las condiciones de este experimento se puede utilizar la boquilla AIC 11003VS o la XRC 11003VS para aplicar paraquat y no habrá diferencia en el control de maleza. Esto se atribuye a que ambas boquillas tuvieron una buena cobertura del follaje de la maleza al usar $250 \mathrm{~L} / \mathrm{ha}$ de agua, lo que causó una buena cobertura del follaje, factor indispensable para un buen control de malezas con paraquat que es un herbicida de contacto. Posiblemente al reducir el volumen de aplicación de paraquat de $250 \mathrm{~L} / \mathrm{ha}$ a 165 L/ha con AIC 11003VS, se disminuirá el control de malezas, debido a que se reduce el área de cobertura de la vegetación (Ramsdale y Messersmitn 2001.

Al bajar la dosis de 500 a $375 \mathrm{~g} / \mathrm{ha}$ de ingrediente activo (equivalente a $75 \%$ de la dosis recomendada), no hubo diferencia estadística $(\mathrm{P} \leq 0.05)$ a los 3 días después de la aplicación. Sin embargo, a los 8 y 15 días hubo una menos control de la maleza al bajar la dosis (Figura 5). El control a los 8 días depués de la aplicación fue de 70 y $75 \%$, con dosis de 500 y 375 $\mathrm{g} /$ ha de ingrediente activo, respectivamente, y a los 15 días fue 48 y $42 \%$, respectivamente. Esto indica que si reducimos la dosis del herbicida también dismuirá su control sobre la maleza. Posiblemente, esto se debe a que a $\operatorname{los} 8$ y 15 días después de la aplicación las plantas que no fueron matadas empiezan a rebrotar y en la evaluación se traduce a menor control.

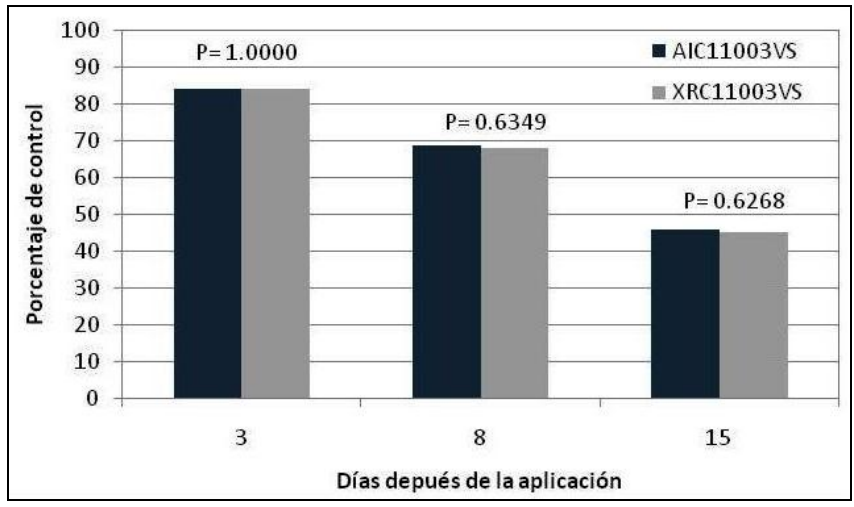

Figura 4. Porcentaje de control de Digitaria spp. con las boquillas AIC11003VS (aire inducido) y XRC11003VS (amplio espectro), a los 3, 8 y 15 días después de la aplicación del paraquat. Los valores sobre las barras indican la probabilidad en cada evaluación.

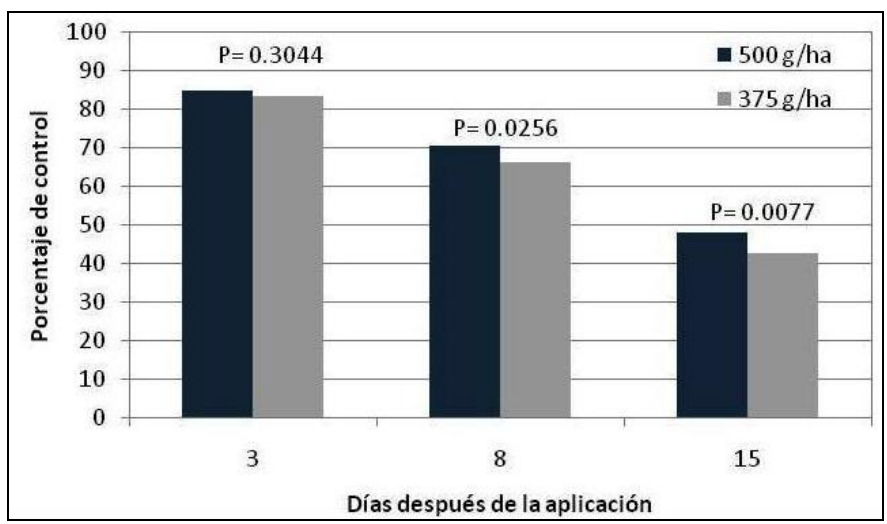

Figura 5. Porcentaje de control de Digitaria spp. con 500 y $375 \mathrm{~g} / \mathrm{ha}$ de ingrediente activo de paraquat. Los valores sobre las barras indican la probabilidad en cada evaluación. 


\section{Conclusiones}

La boquilla AI11003VS tiene gotas más grandes que las de XR11003VS, pero esto no influyó en el control de malezas con glifosato ni paraquat.

Al aplicar glifosato, las boquillas AI11003VS y XR11003VS no presentaron diferencia en el control del pasto estrella.

Al aplicar paraquat con las boquillas AIC11003VS y XRC11003VS no hubo diferencia en el control de Digitaria spp.

$\mathrm{Al}$ disminuir la dosis de glifosato de $890 \mathrm{~g} / \mathrm{ha} a$ $668 \mathrm{~g} / \mathrm{ha}$ de ingrediente activo, se redujo el control del pasto estrella.

Disminuir la dosis de paraquat de $500 \mathrm{~g} / \mathrm{ha}$ a 375 $\mathrm{g} / \mathrm{ha}$ de ingrediente activo se redujo el control sobre Digitaria spp.

Agradecimiento. Muchas gracias al Dr. Noha Holah, Post doc research associate en el Departamento de Fitopatología, Iowa State University, Ames, Iowa, por determinar la cobertura de las gotas de agua en el papel hidrosensible.

\section{Literatura Citada}

Doll, J. 1981. Factores que condicionan la eficiencia de los herbicidas; Cali, Colombia CIAT. 9 p.

De la Cruz, R. 1986. Técnicas de investigación en malezas. In: J. Pinochet y G. von Lindeman (ed) Seminario taller de malezas. CATIE, Panamá. p 25-26.

Feng, P.C.C., Chiu, T. y Ryerse, J.S. 2003. Droplet size affects glyphosate retention, absorption, and translocation in corn. Weed Science 51:443-448.

Gómez Vargas, J.M., A. Pitty y J.M. Miselem. 2006. Efecto del $\mathrm{pH}$ del Agua en la Efectividad de los Herbicidas Glifosato, Fluazifop-p-butil y Bentazon. Ceiba 47(12):19-23.
Hatterman-Valenti, H.M; Pitty, A. and M.D.K. Owen. 2006. Effect of environment on giant foxtail (Setaria faberi) leaf wax and fluazifop-P absorption. Weed Science 54:607-614.

Jones, J., Hanks, J. y Wills, G. 2002. Effect of different nozzle types on drift and efficacy of Roundup Ultra; Stoneville, Mississippi. 2 p.

Knoche, M. 1994. Effect of droplet size and carrier volume on performance of foliage-applied herbicides. Crop Protection 13(3):163-178

Labrada, R. Casely, J. y Parker, C. 1996. Manejo de malezas para países en desarrollo; Roma, Italia FAO. 204 p.

Matthews, G. 1979. Pesticide application methods. Longman, Inc. New York, N.Y. p 306.

Ramsdale, B.K. y C.G. Messersmith. 2001. Drift-reducing nozzles effects on herbicide performance. Weed Technology 15(3):453-460.

Rosses, M. y Urrutia, C. 1999. Estudio sobre la caracterización, producción, análisis de mercado y rentabilidad del cultivo de cebolla; Managua, Nicaragua IICA. $17 \mathrm{p}$.

Spraying Systems Co. 2004. Guía del usuario de boquillas de pulverización; Wheaton, Illinois 60189-7900 USA.

Steel, R.G.D. y J.H. Torrie. 1980. Principles and procedures of statistics, a biometrical approach. 2da ed. McGrawHill, New York, Estados Unidos. p 236.

Tharrington, W. y Tate, R. 1976. Algunas consideraciones respecto a las boquillas para uso agrícola. Iowa. $179 \mathrm{p}$.

Thornhill, E. y Matthews, G. 1994. Equipo de aplicación de pesticidas para uso en la agricultura, volumen 2. Reino Unido. p 23 y 95.

Venturelli, L., Tesouro, O., Masiá, G. y Fuica, A. 2006. Cobertura y respuesta biológica del Glifosato ante la utilización de distintas pastillas de pulverización; Buenos Aires, Argentina INTA. 2 p.

Zhou, J., B. Tao y C.G. Messersmith. 2006. Soil dust reduces glyphosate efficacy. Weed Science 54(6):11321136.

Recibido para publicación el 27 de noviembre de 2012. Aceptado para publicación el 15 de abril de 2013. 